\title{
Proximal Triplane Tibial Fracture in a 6-Year-Old Girl
}

\author{
Ziyad AlShaqsi, Sara Alhabsi, Yumna Alnaabi, and Yaqoub Almufargi
}

\section{ABSTRACT}

Proximal tibial fractures are very rare. Our case is about a six-year-old girl presented with proximal tibial triplane fracture associated with proximal and distal fibula and distal tibial fractures, as a result of a fall of a cement wall on her right knee. A radiograph and computerized tomography (CT) scan were reported as Salter-Harris type III fracture. She was treated by an open reduction and internal fixation by a screw and Kirschner wires. She was followed up for $\mathbf{1 2}$ months and showed an excellent knee outcome and her knee range of motion was $10-130^{\circ}$. Anatomical reduction and stable fixation are necessary to prevent future pain, deformity and arthritis. The case demonstrates the efficacy of using an open reduction and internal fixation by a screw and Kirschner wires in treating children with triplane proximal tibial fracture. This type of fracture is not frequently seen and a right diagnosis leads to better management, which could prevent future complications.

Keywords: child, proximal tibia, Salter-Harris, tibial fracture, triplane.

Submitted : January 31, 2021

Published : February 10, 2021

ISSN: 2593-8339

DOI: $10.24018 /$ ejmed.2021.3.1.701

\section{Ziyad AlShaqsi *}

Department of Orthopedic, Armed

Forces Hospital, Oman.

(e-mail: z911hassan@gmail.com)

Sara Alhabsi

Sultan Qaboos University, Oman

(e-mail: sara2211@hotmail.com)

Yumna Alnaabi

Sultan Qaboos University, Oman

(e-mail: Yumna@hotmail.com)

Yaqoub Almufargi

Department of Orthopeadic, Armed

Forces Hospital, Oman.

(e-mail: yalmufargi@gmail.com)

*Corresponding Author

\section{INTRODUCTION}

Proximal tibial fractures are rare and present less than $1 \%$ of pediatric fractures. This could be due to the surrounding fibrous structures supporting the knee [1], [2]. They could be caused by either direct trauma or indirect trauma to the knee [3]. Salter-Harris classification is used to classify physical injuries in children [4]. Proximal triplane tibial fractures (Salter-Harris type III) are rare intra-articular fractures [1]. A comprehensive history and physical evaluation are crucial to decide on the appropriate management and to determine the presence or absence of poor prognostic markers such as high-energy trauma and high-grade Salter-Harris injury (type III or IV) [5]. We report a rare case of proximal triplane tibial fracture (SalterHarris type III) associated with distal tibial fracture and proximal and distal fibular fractures. Despite the poor prognosis of this type of fractures as the physis contributes to the length of the limb and the growth of the tibia, our patient did well.

\section{AIM}

To demonstrate the efficacy of using an open reduction and internal fixation by a screw and Kirschner wires in treating children with triplane proximal tibial fracture.

\section{CASE History}

A 6-year-old girl presented to the emergency department (ED) after a history of a fall of a cement wall on her right lower limb with two contused injuries. Her physical examination showed that she has two contused wounds in the lower limb and valgus deformity with no neurovascular abnormality. Plain radiography showed proximal tibia fracture classified as Salter-Harris type III and a right distal tibial fracture with valgus and recurvatum angulation (Fig. 1), as well as a segmental fibular fracture. Computed tomography (CT) scan was reported as a comminuted fracture of the proximal physis and epiphysis of the right tibia (triplane) with posterior displacement of the fractured epiphysis and multiple intra-articular bony fragments (Salter-Harries type III) (Fig. 2) and lipohemoarthrosis. A bony alignment was done, and an above-knee back slap was applied as initial management in the ED.

She was operated on the next day. Under general anesthesia, open reduction of the proximal tibial fragment was made, and the tibial spine was fixed with a fiber wire. Fixation of the medial tibia condyle fracture was done with a size $3 \times 34 \mathrm{~mm}$ partially threaded cannulated screw with a washer. The medial meniscus was sutured to the capsule with a fiber wire suture. The distal tibial fracture was reduced with a closed method under image guidance and was fixed with two lateral and one medial two $\mathrm{mm}$ Kirschner wires (K-wires) (Fig. 3). An above knee cast was applied for six weeks to ensure good consolidation and 
avoid secondary displacement of the fracture. She was mobilized non-weight-bearing. No postoperative complications were noted, and she was discharged on day three with analgesics and home instructions. The K-wires were removed after 4 weeks and the screw after 12 months (Fig. 4). An intensive physiotherapy program was started directly after the removal of the screw, to improve her right knee extension. She had no pain now and has a range of motion of about $10-130^{\circ}$.
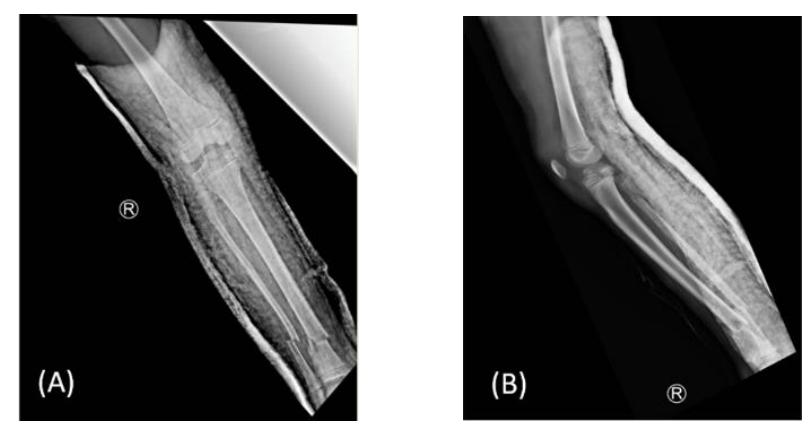

Fig. 1. (a) anteroposterior $X$ ray of the right knee and (b) lateral view of the right knee Salter-Harris type III.
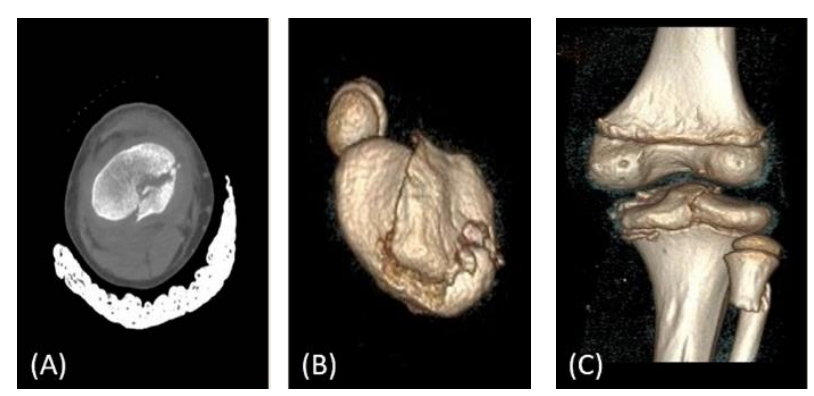

Fig. 2. (a) CT scan of right knee and (b), (c) 3D picture of the right knee preoperation.
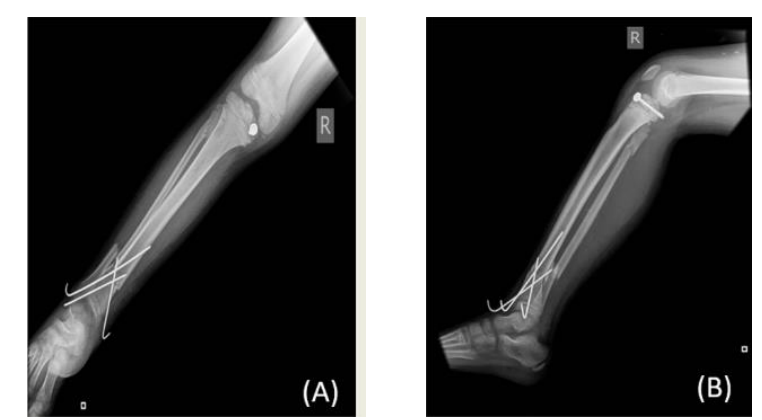

Fig. 3. (a) anteroposterior X-ray of the right knee and distal tibia and (b) lateral view after operation.
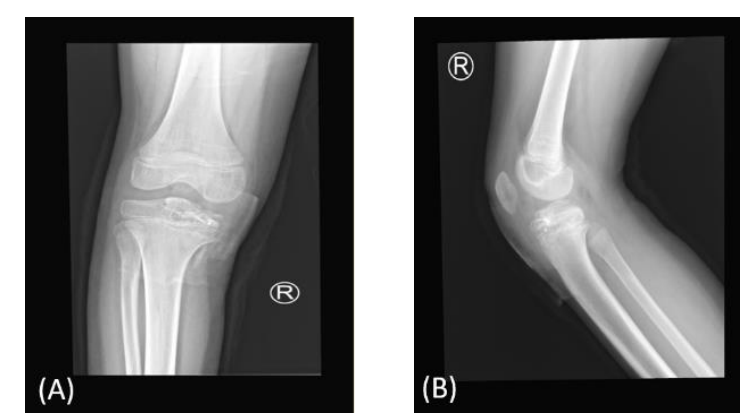

Fig. 4. (a) anteroposterior and (b) lateral view of the right knee after 11 months of follow up.

\section{DISCUSSION}

The reason behind the rarity of proximal triplane tibial fractures is the symmetrical closure of the physis. In fact, no clear idea of how the closure happens, in contrast to the distal tibial physis closure. Haines et al. explained the physeal closure in the proximal tibial physis that starts anteromedially and proceeds posterolaterally [6].

Different mechanism can result in triplane proximal tibial fracture. In our case, the girl was subjected to a high energy trauma in the form of a fall of a heavy weight cement wall on her right lower limb. Othman et al. reported a similar fracture in a 16-year-old boy who had distal femur and proximal tibia fractures due to motor vehicle collision while the knee in a valgus and hyperextension position [7].

If the proximal tibial fracture is suspected, as in the current case, an anterior-posterior (AP) and lateral plain radiographs of the lower limb, including both knee and ankle, should be obtained. In the present case, the plain radiograph revealed proximal tibial fracture with angulation associated with distal tibial fracture and proximal and distal fibular fractures. CT scan, and magnetic resonance imaging (MRI) are important in case of complicated fractures [2]. Dvonch et al. reported a case of (ipsilateral distal femoral and proximal tibial epiphyseal growth plate injuries) where they missed the diagnosis of Salter-Harris type III because their attention was towards the femoral injury [8]. The CT scan of this child was reported as a comminuted fracture of the proximal physis and epiphysis of the right tibia (SalterHarris type III).

An infrequent worrisome complication is that the posterior displacement of the fractured proximal physis can injure the popliteal artery, which eventually may lead to ischemia if not managed properly [2]. As Salter-Harris type III fractures involve both the physis and epiphysis, it is documented that $25-28 \%$ of the length of the extremity and approximately around $57-60 \%$ of the growth of the tibia is contributed to by the proximal tibial physis [9]. Consequently, injuries to physis and epiphyses can lead to growth disturbances [2]. The most common long-term complications are limb shortening and coronal deformity [10]. One month after the injury, the K-wires removed and eleven months follow-up after injury for the present case revealed no postoperative complication, the patient is doing well, and removal of the right proximal tibial screw was done in the same appointment. The patient was also referred for intensive physiotherapy to improve the range of motion and to strengthen the muscles around the knee joint. This type of fractures not frequently seen, and a right diagnosis could lead to excellent management, which could prevent future complications such as limb shortening, deformity and osteoarthritis.

\section{CONCLUSION}

Proximal tibial triplane fracture is a rare pediatric fracture. It is caused by high energy and involves the phases, joint and metaphysis. Anatomical reduction and stabilization are necessary to prevent future pain, deformity and arthritis. 


\section{RECOMMENDATIONS}

Radiographic follow-up will help prevent leg length difference, angular deformity or early arthritis, which may happen because the injury involves the growth plate and the joint.

\section{FINANCIAL SUPPORT}

This study did not receive any specific grant from funding agencies in the public, commercial, or not-for-profit sectors.

\section{ETHICAL APPROVAL AND DEClARATION OF PATIENT \\ CONSENT}

The author certifies that he has obtained all appropriate patient consent forms. In the form, the patient parents have given their consent for the images and other clinical information to be reported in the journal. The parents understand that the name and initials will not be published, and due efforts will be made to conceal the identity, but anonymity cannot be guaranteed.

\section{REFERENCES}

[1] Vrettakos A. N., Evaggelidis D. C., Kyrkos M. J., Tsatsos A. V., Nenopoulos A., Beslikas T. Lower limb deformity following proximal tibia physeal injury: long-term follow-up. Journal of Orthopaedics and Traumatology. 2012;13(1):7-11.

[2] Caillon, F., Rigault, P., Padovani, J. P., Janklevicz, P., Langlais, J. Les traumatismes de l'extrémité supérieure du tibia chez l'enfant: a l'exclusion des fractures des épines tibiales. Chirurgie pédiatrique (Paris), 31(6) (1990), 322-332.

[3] Little, R. M., \& Milewski, M. D. Physeal fractures about the knee. Current reviews in musculoskeletal medicine, 9(4) (2016), 478-486.

[4] Jawetz, S. T., Shah, P. H., \& Potter, H. G. Imaging of physeal injury: overuse. Sports Health, 7(2) (2015), 142-153.

[5] Dabash, S., Prabhakar, G., Potter, E., Thabet, A. M., Abdelgawad, A., \& Heinrich, S. Management of growth arrest: Current practice and future directions. Journal of clinical orthopaedics and trauma, 9 (2018), S58-S66.

[6] Haines, R. W. The histology of epiphyseal union in mammals. Journal of anatomy, 120(Pt 1) (1975), 1.

[7] Othman, Y., Hassini, L., Fekih, A., Aloui, I., \& Abid, A. Uncommon Floating Knee in a Teenager: A Case Report of Ipsilateral Physeal Fractures in Distal Femur and Proximal Tibia. Journal of orthopaedic case reports, 7(2) (2017), 90.

[8] Dvonch, V. M., \& Bunch, W. H. Pattern of closure of the proximal femoral and tibial epiphyses in man. Journal of pediatric orthopedics, 3(4) (1983), 498-501.

[9] Pane D. N., Fikri M. E. L., Ritonga H. M. Epiphyseal Growth Plate Fracture. Journal of Chemical Information and Modeling. 2018;53. 462-469

[10] Gautier, E., Ziran, B. H., Egger, B., Slongo, T., \& Jakob, R. P. Growth disturbances after injuries of the proximal tibial epiphysis. Archives of Orthopaedic and Trauma Surgery, 118(1-2) (1998), 3741. 